\title{
Numerical Simulation of Rotating Turbulent Channel Flow by the Vortex in Cell Method
}

\author{
Tomomi Uchiyama ${ }^{1,{ }^{*}}$, Hirotaka Hamada ${ }^{2}$ and Tomohiro Degawa ${ }^{3}$ \\ ${ }^{I}$ EcoTopia Science Institute, Nagoya University, Furo-cho, Chikusa-ku, Nagoya 464-8603, Japan \\ ${ }^{2}$ Hitachi Solutions, Ltd., 4-12-7, Higashishinagawa, Shinagawa-ku, Tokyo 140-0002, Japan \\ ${ }^{3}$ Numazu National College of Technology, 3600, Ooka, Numazu, Shizuoka 410-8501, Japan
}

\begin{abstract}
Direct numerical simulation (DNS) of a fully-developed turbulent flow in a channel rotating around the spanwise axis is performed. A vortex in cell (VIC) method, of which numerical accuracy was successfully improved by the authors in their prior study, is applied to the DNS. The Reynolds number based on the friction velocity and the channel half width is 171 , and the nondimensional rotation number defined with the channel angular velocity and width is 2.1 . The simulated turbulence statistics, such as the mean velocity and the Reynolds shear stress, are favorably compared with the existing DNS results. The simulation can analyze the disappearance of the streak structures near the suction-wall due to the channel rotation. It also captures the secondary flow of the Taylor-Görtler vortex pattern near the pressure-wall. These simulation results demonstrate that the VIC method improved by the authors is indeed applicable to the DNS of turbulent flows in rotating channel.
\end{abstract}

Keywords: Direct numerical simulation, Rotating turbulent channel flow, Taylor-Görtler vortex, Turbulence, Vortex in cell method, Vortical structure.

\section{INTRODUCTION}

Vortex in cell (VIC) method is one of the numerical computation methods of incompressible flows [1]. It is classified as vortex methods, which simulate the time evolution of the flow by solving a vorticity equation. The VIC method discretizes the vorticity field into vortex elements and computes the time evolution of the flow by tracing the convection of each vortex element using the Lagrangian approach. The Lagrangian calculation markedly reduces numerical diffusion and also improves numerical stability. Thus, the VIC method is eminently suitable for direct numerical simulation (DNS) of turbulent flows. Various simulation results have been reported. Cottet and Poncet [2] applied the VIC method for the wake simulation of a circular cylinder, and captured the streamwise vortices occurring behind the cylinder. Cocle et al. [3] analyzed the behavior of two vortex system near a solid wall, and made clear the interaction between two counter-rotating vortices and the eddies induced in the vicinity of the wall. Chatelain et al. [4] simulated trailing edge vortices, and visualized the unsteady phenomena caused by disturbances. These studies are concerned with time-developing free shear flows, which are dominated by the convection of large-scale eddy. But the VIC method has not been applied to the turbulent flow bounded by solid walls. Thus, the authors [5] performed the DNS of a

*Address correspondence to this author at the EcoTopia Science Institute, Nagoya University, Furo-cho, Chikusa-ku, Nagoya 464-8603, Japan;

Tel: +81-52-789-5187;

E-mail: uchiyama@is.nagoya-u.ac.jp turbulent channel flow, which is a representative example of the wall turbulent flows. The Reynolds number based on the friction velocity and the channel half width is 180 . They applied an improved VIC method, of which computational accuracy was successfully heightened by them [5]. The DNS highlighted that the turbulence statistics, such as the mean velocity, the Reynolds shear stress, and the velocity and vorticity fluctuations, are in good agreement with the existing DNS results. It also indicated that the organized flow structures, such as streaks and streamwise vortices appearing in the near wall region, are favorably captured. These demonstrated the validity of the DNS for turbulent channel flows performed with the VIC method improved by the authors.

Incompressible flows in rotating fields have been extensively studied by a number of researches [6-9] to understand air currents in the atmosphere and to grasp turbomachinery flows. Turbulent flows in channel rotating around the spanwise axis were investigated by the LES $[10,11]$ and the DNS [12, 13], and the effects of the Coriolis force on the flow stability and turbulent structures were explored. The VIC method is expected to be useful for the DNS of the rotating turbulent channel flows. But such DNS has not been carried out.

In this study, the DNS of a rotating turbulent channel flow is performed with the VIC method, of which computational accuracy was improved by the authors [5]. The Reynolds number based on the friction velocity and the channel half width is 171 , while the nondimensional rotation number defined with the channel angular velocity and width is 2.1 . The simulation highlights that the effect of the channel rotation on the turbulence statistics is favorably analyzed, dem- 


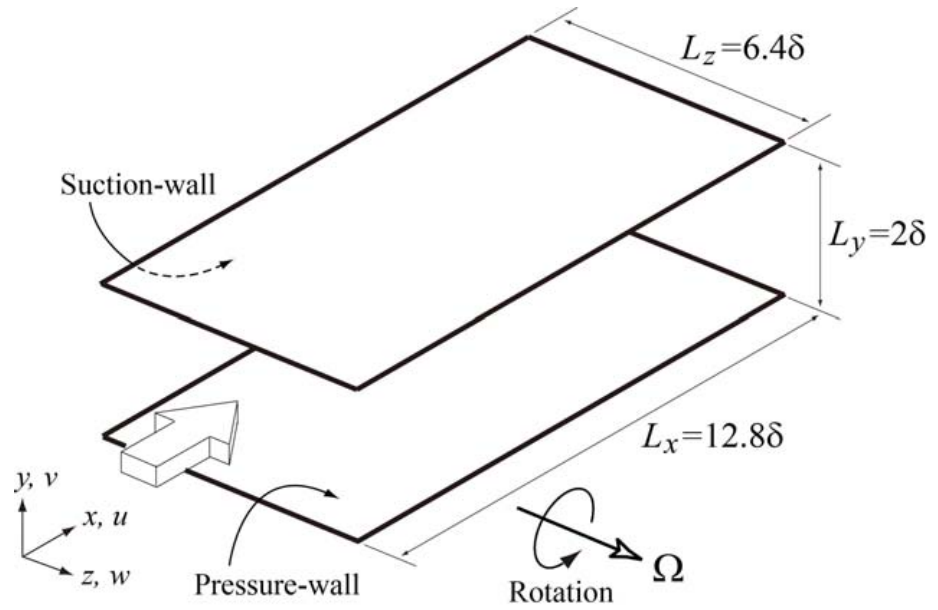

Fig. (1). Rotating channel and coordinate system.

onstrating the validity of the DNS for the rotating turbulent channel flows by the authors' VIC method.

\section{VORTEX IN CELL METHOD}

Let us consider an incompressible fully-developed turbulent flow between two infinite parallel plates in spanwise rotation with a constant angular velocity $\Omega$ about the $z$-axis in a Cartesian coordinate system, as shown schematically in Fig. (1). The governing equations are the Navier-Stokes and continuity equations written in a reference frame rotating with the plates. The vorticity equation is given by

$$
\frac{\partial \omega}{\partial t}+\nabla \cdot(\omega \mathbf{u})=\nabla \cdot(\mathbf{u} \omega)+v \nabla^{2} \omega+(2 \Omega \cdot \nabla) \mathbf{u}
$$

where $\omega(=\nabla \times \boldsymbol{u})$ is the vorticity.

According to the Helmholtz theorem, the velocity $\boldsymbol{u}$ is the sum of the curl of a vector potential $\psi$ and the gradient of a scalar potential $\phi$. The velocity $\boldsymbol{u}$ is thus expressed as

$\mathbf{u}=\nabla \times \psi+\nabla \phi$

When $\psi$ is postulated to be solenoidal or $\nabla \cdot \psi=0$, the curl of Eq. (2) yields the vector Poisson equation for $\psi$ :

$\nabla^{2} \psi=-\omega$

Substituting Eq. (2) into the continuity equation and rewriting the resultant equation, the Laplace equation for $\phi$ is obtained:

$\nabla^{2} \phi=0$

Once $\psi$ and $\phi$ have been computed from Eqs. (3) and (4) respectively, the velocity $\boldsymbol{u}$ is calculated from Eq. (2). The vorticity $\boldsymbol{\omega}$ in Eq. (3) is estimated from Eq. (1). The vortex in cell (VIC) method discretizes the vorticity field into vortex elements, and calculates the distribution of $\omega$ by tracing the convection of each vortex element.

It is postulated that the position vector and vorticity for the vortex element $p$ are $\boldsymbol{x}_{p}=\left(x_{p}, y_{p}, z_{p}\right)$ and $\omega_{p}$ respectively. The Lagrangian form of the vorticity equation, Eq. (1), is written as follows [14]: $\frac{\mathrm{d} \boldsymbol{x}_{p}}{\mathrm{~d} t}=\mathbf{u}\left(\boldsymbol{x}_{p}\right)$

$\frac{\mathrm{d} \omega_{p}}{\mathrm{~d} t}=\nabla \cdot\left(\mathbf{u}\left(\mathbf{x}_{p}\right) \omega\left(\mathbf{x}_{p}\right)\right)+v \nabla^{2} \omega\left(\mathbf{x}_{p}\right)+(2 \Omega \cdot \nabla) \mathbf{u}\left(\mathbf{x}_{p}\right)$

When the position and vorticity of a vortex element are known at time $t$, the values at $t+\Delta t$ are computed from Eqs. (5) and (6). In the VIC method, the flow field is divided into computational grid cells to define $\psi, \phi$ and $\omega$ on the grids. If $\omega$ is defined at a position $\boldsymbol{x}_{g}=\left(x_{g}, y_{g}, z_{g}\right)$, the vorticity $\omega$ is assigned to $\boldsymbol{x}_{g}$, or a vortex element with vorticity $\omega$ is redistributed onto $\boldsymbol{x}_{g}$ :

$\omega=\sum_{p}^{N_{v}} \omega_{p} W\left(\frac{x_{g}-x_{p}}{h_{x}}\right) W\left(\frac{y_{g}-y_{p}}{h_{y}}\right) W\left(\frac{z_{g}-z_{p}}{h_{z}}\right)$

where $N_{v}$ is the number of vortex elements, and $h_{i}(i=x, y, z)$ is the grid width. For the redistribution function $W$, various forms are presented [14]. To suppress the numerical dissipation, a high-order scheme, which preserves the three first moments of the distribution, twice continuously differentiable and symmetric, is employed for $W$ :

$W(\varepsilon)=\left\{\begin{array}{cc}1-2.5 \varepsilon^{2}+1.5|\varepsilon|^{3} & |\varepsilon|<1 \\ 0.5(2-|\varepsilon|)^{2}(1-|\varepsilon|) & 1 \leq|\varepsilon| \leq 2 \\ 0 & |\varepsilon|>2\end{array}\right.$

\section{IMPROVEMENTS OF VIC METHOD}

\subsection{Discretization by Staggered Grid}

For incompressible flow simulations, the MAC and SMAC methods solve the Poisson equation, which is derived from the equation for pressure gradient and the continuity equation. These methods employ a staggered grid to ensure consistency among the discretized equations, and to prevent numerical oscillations of the solution. The staggered grid would appear to be useful for discretizing the Poisson equation for $\psi$ and the Laplace equation for $\phi$, which are derived 


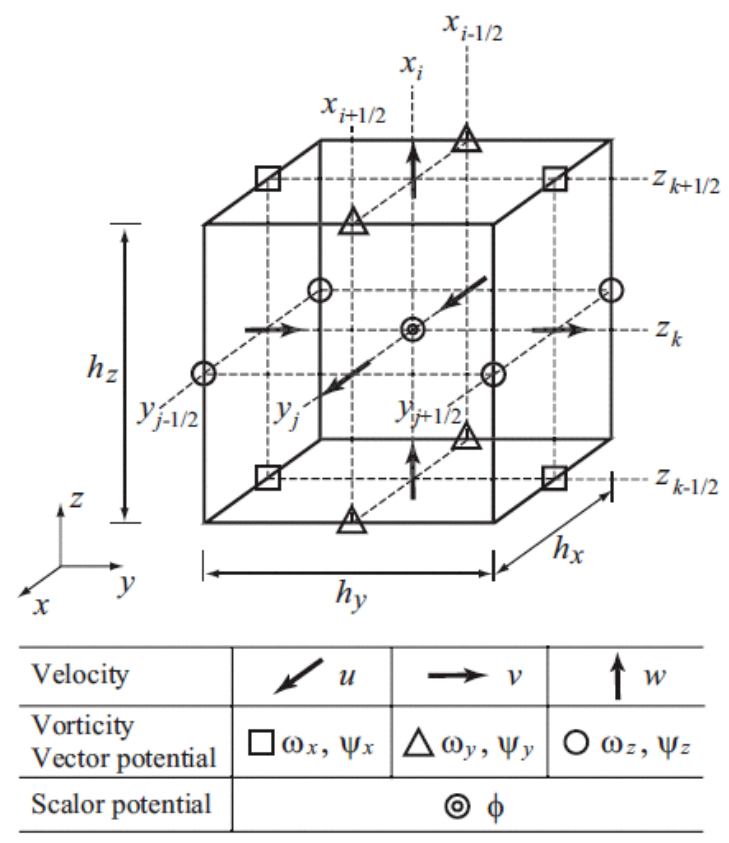

Fig. (2). Staggered grid.

in the VIC method. However, staggered grids are not readily accommodated in the existing VIC method.

The authors proposed a VIC method using a staggered grid in their prior study [5]. Fig. (2) shows the arrangement of the variables in the grid. The scalar potential $\phi$ and the velocity $\boldsymbol{u}$ are defined at the center and sides of the grid, respectively. The vorticity $\omega$ and the vector potential $\psi$ are defined on the edges.

\subsection{Correction of Vorticity Field}

In the VIC method, the vorticity field is discretized into vortex elements, and the field is expressed by superimposing the vorticity distributions around each vortex element. This superposition is performed by Eq. (7). The resulting vorticity field $\omega_{r}$ does not necessarily satisfy the solenoidal condition [14]. Denoting the vorticity satisfying this condition by $\omega_{s}$ $(=\nabla \times \mathbf{u}), \omega_{r}$ is expressed as [3]

$\omega_{r}=\nabla F+\omega_{s}$

where $F$ is a scalar function. Equation (9) corresponds to the Helmholtz decomposition of $\omega_{r}$.

Taking the divergence of Eq. (9), the Poisson equation for $F$ is obtained:

$\nabla^{2} F=\nabla \cdot \omega_{r}$

Calculating $F$ from Eq. (10) and substituted into Eq. (9) gives the recalculated vorticity $\omega_{s}$ [3]. This correction for vorticity needs to solve the Poisson equation, which increases the computational time. To reduce this additional cost, the authors [5] have proposed a simplified correction method.

The uncorrected vorticity, $\omega_{r}$, is linked to $\psi_{r}$ through Eq. (3). Taking the divergence of Eq. (3) and substituting Eq. (10) into the resultant equation, the following relations are obtained:

$$
\begin{aligned}
\nabla^{2}\left(\nabla \cdot \psi_{r}\right) & =-\nabla \cdot \omega_{r} \\
& =-\nabla^{2} F
\end{aligned}
$$

Unlike the assumption for the solenoidal condition of $\psi$, the following equation for a non-solenoidal vorticity is derived from Eq. (11).

$\nabla \cdot \psi_{r}=-F$

Using $\omega_{r}$ to calculate $\psi_{r}$ from Eq. (3), and determining $\phi_{r}$ from Eq. (4), the curl of $\mathbf{u}_{r}$ transforms as follows:

$$
\begin{aligned}
\nabla \times \mathbf{u}_{r} & =\nabla \times\left(\nabla \phi_{r}+\nabla \times \psi_{r}\right) \\
& =\nabla\left(\nabla \cdot \psi_{r}\right)-\nabla^{2} \psi_{r} \\
& =-\nabla F+\omega_{r} \\
& =\omega_{s}
\end{aligned}
$$

Equation (13) demonstrates that the curl of the velocity $\mathbf{u}_{r}$ calculated from $\omega_{r}$ yields a vorticity $\omega_{s}$ that satisfies the solenoidal condition. If the vorticity is recalculated by Eq. (13), or the vorticity is corrected immediately after calculating the velocity by Eq. (2), the discretization error in the vorticity is completely removed and the flow dynamics are accurately simulated without solving the Poisson equation [Eq. (10)]. It should be noted that the staggered grid is required for rendering the transformation in Eq. (13) applicable to the corresponding discretized equations.

\subsection{Simulation Procedure}

Given the flow at time $t$, the flow at $t+\Delta t$ is simulated by the following procedure:

1. Calculate the change in the strength of each vortex element, or calculate the vorticity $\omega_{p}$ from Eq. (6).

2. Calculate the convection of each vortex element, or calculate the position $\boldsymbol{x}_{p}$ from Eq. (5). 


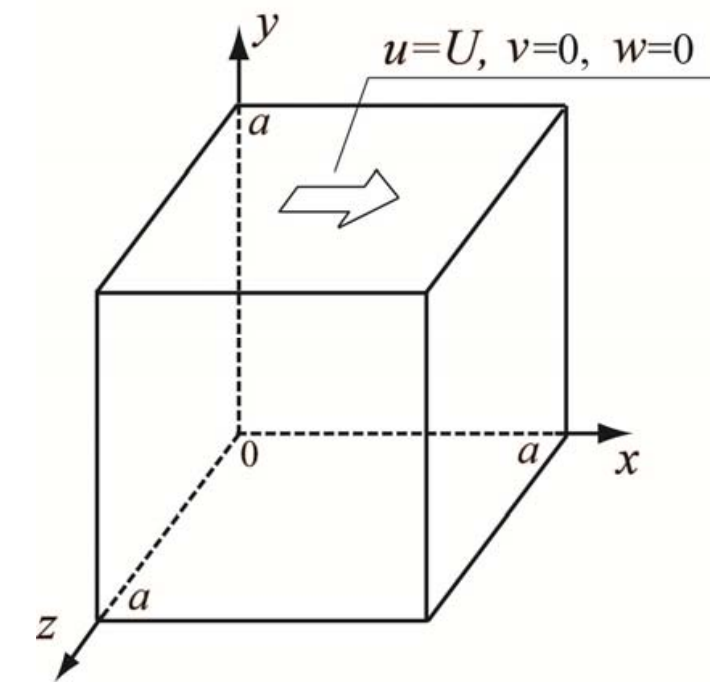

Fig. (3). Cubic cavity and coordinate system.
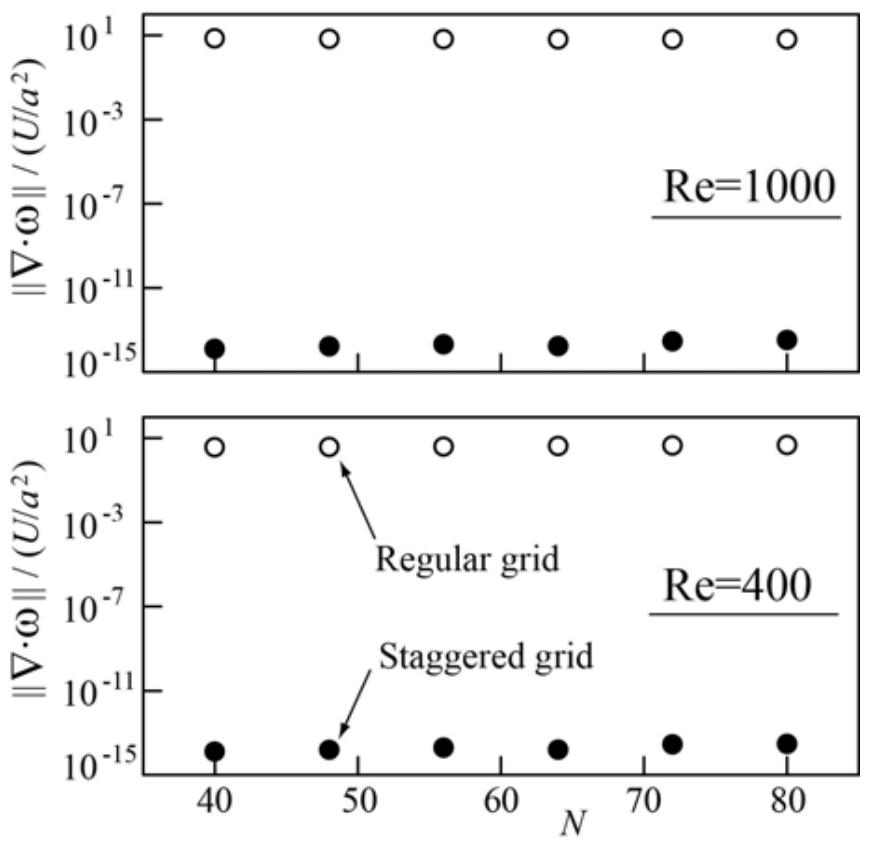

Fig. (4). Rms for divergence of vorticity in cubic cavity flow at $\mathrm{Re}=400$ and 1000 .

3. Redistribute the vortex element onto the grids, or calculate the vorticity $\omega$ on the grids by Eq. (7).

4. Calculate the vector potential $\psi$ from Eq. (3).

5. Calculate the scalar potential $\phi$ from Eq. (4).

6. Calculate the velocity $\boldsymbol{u}$ from Eq. (2).

7. Correct the vorticity, or calculate the corrected vorticity from the curl of $\boldsymbol{u}$.

\subsection{Application to Cavity Flow Simulation}

To demonstrate the applicability of the abovementioned improved VIC method, the flows in a cubic cavity are simulated [5]. A comparison simulation using regular grids is also conducted, in which the correction of the vorticity by Eq. (13) is not employed.

Fig. (3) shows the computational domain and the coordinate system. The side length of the cavity is $a$. The upper wall $(y / a=1)$ moves in the $x$-direction with velocity $U$. The Reynolds number $\operatorname{Re}$ is defined as $a U / v$. The domain is divided into uniform grids, and the time increment $U \Delta t / a$ is 0.01. The non-slip condition is imposed on the walls. Equation (3) is solved by the SOR method. The second-order Adams-Bashforth method is used for the Lagrangian calculation of Eqs. (5) and (6).

Calculating the divergence of the vorticity on each grid point at $\mathrm{Re}=400$ and 1000 , the root-mean-square $\|\nabla \cdot \omega\|$ varies as shown in Fig. (4), where $N$ is the number of grids along one axis of the cubic cavity. In the simulation using the staggered grids with the vorticity correction method, the vorticity field sufficiently satisfies the solenoidal condition. One can find the applicability of the authors' VIC method. 
Table 1. Computational Conditions

\begin{tabular}{|c|c|c|c|}
\hline Coordinate & $\begin{array}{c}\text { Streamwise } \\
\qquad x\end{array}$ & $\begin{array}{c}\text { Wall-normal } \\
y\end{array}$ & $\begin{array}{c}\text { Spanwise } \\
z\end{array}$ \\
\hline Domain length $L$ & $12.8 \delta$ & $2 \delta$ & $6.4 \delta$ \\
\hline$L^{+}$ & 2191 & 342 & 1096 \\
\hline Grid number $N$ & 256 & 512 & 256 \\
\hline Grid size $u_{\tau} h / v$ & 8.56 & 0.669 & 4.28 \\
\hline Time increment $\Delta t^{+}$ & & 0.018 & \\
\hline Reynolds number $\mathrm{Re}_{\tau}$ & & 171 & \\
\hline Rotation number $\mathrm{Ro}_{\tau}$ & & 2.1 & \\
\hline
\end{tabular}

\section{COMPUTATIONAL CONDITIONS}

The fully-developed turbulent flow between two infinite parallel plates in spanwise rotation is simulated. Note that $x$, $y$ and $z$ imply the streamwise, wall-normal and spanwise directions respectively, and that $u, v$ and $w$ are the velocity components in the $x, y$ and $z$ directions respectively, as shown in Fig. (1). The channel width $L_{y}$ is $2 \delta$, while the streamwise period $L_{x}$ and the spanwise one $L_{z}$ are set at $12.8 \delta$ and $6.4 \delta$ respectively. The computational domain is divided into $256 \times 512 \times 256$ uniform grids. The boundaries at $y / \delta=0$ and 2 correspond to the pressure- and suctionwalls respectively. The computational conditions are listed in Table $\mathbf{1}$.

A non-slip condition is imposed on the walls, while a periodic boundary condition is assumed in the $x$ - and $z$ directions. The velocity normal to the wall is zero, and the relation $\nabla \cdot \psi=0$ should be satisfied on the wall. These imply that the tangential component of $\psi$ and the normal derivative of the normal component of $\psi$ are zero. The non-slip condition on the walls is realized by imposing the velocity on the grid points located outside of the wall. The boundary condition of $\omega$ on the wall is given by the curl of the velocity. The boundary conditions are summarized as:

$\mathbf{u}=0$

$\psi_{x}=0, \psi_{z}=0, \frac{\partial \psi_{y}}{\partial y}=0$

$\omega_{x}=\frac{\partial w}{\partial y}, \omega_{y}=0, \omega_{z}=-\frac{\partial u}{\partial y}$

where $\omega_{x}$ and $\omega_{z}$ are estimated with a second-order central difference scheme by using the velocities on the grid points located inside and outside of the wall.

The Poisson equation, Eq. (3), is solved with the aid of the fast Fourier transform in the streamwise $(x)$ and spanwise (z) directions. The mean flow is driven by imposing a bulk velocity $u_{m}$. As the scalar potential satisfies the relation $\phi=u_{m} x$, the velocity $\boldsymbol{u}$ is calculated from the following equation: $\mathbf{u}=\nabla \times \psi+u_{m}$

First, the flow in the non-rotating channel has been calculated when the Reynolds number $\operatorname{Re}\left(=2 u_{m} \delta / v\right)$ is set at 5626. It was confirmed that the Reynolds number $\operatorname{Re}_{\tau 0}$ $\left(=u_{\tau 0} \delta / v\right)$, based on the friction velocity $u_{\tau 0}$ and $\delta$, is 180. This agreed with the DNS of Kim et al. [15]. By using this flow field as the initial condition, the present study simulated the flow at the nondimensional rotation number $\operatorname{Ro}_{\tau 0}=2$, where $\operatorname{Ro}_{\tau 0}=2 \Omega \delta / u_{\tau 0}$. The simulation demonstrated that the friction velocity at the pressure-wall $u_{\tau p} / u_{\tau 0}$ is 1.14 and that the friction velocity at the suction-wall $u_{\tau s} / u_{\tau 0}$ is 0.713 . This study defines the friction velocity $u_{\tau}$ by the following equation with reference to the definition of Kristoffersen and Andersson [12] and Grundestam et al. [13].

$u_{\tau}^{2}=\left(u_{\tau p}^{2}+u_{\tau s}^{2}\right) / 2$

In the current simulation, $u_{\tau} / u_{\tau 0}$ is 0.951 , Reynolds number $\operatorname{Re}_{\tau}\left(=u_{\tau} \delta / v\right)$ is 171 , and the nondimensional rotation number $\operatorname{Ro}_{\tau}\left(=2 \Omega \delta / u_{\tau}\right)$ is 2.1 .

The time increment $\Delta t^{+}\left(=\Delta t u_{\tau}^{2} / v\right)$ is set to 0.018 . The computation was executed up to a time of $5100 \mathrm{v} / \mathrm{u}_{\tau}^{2}$ to obtain the fully-developed flow. It was further continued for $965 v / u_{\tau}^{2}$ to calculate the statistical average.

\section{RESULTS AND DISCUSSION}

\subsection{Turbulence Statistics}

The distribution of the mean velocity $\overline{u^{+}}\left(=\bar{u} / u_{\tau}\right)$ is shown in Fig. (5). When compared with the distribution at the non-rotating condition $\left(\mathrm{Ro}_{\tau}=0\right.$ ) obtained by the authors [5], the velocity gradient in the wall-normal direction is larger near the pressure-wall $\left(y^{*}=y / \delta=0\right)$ and lower near 


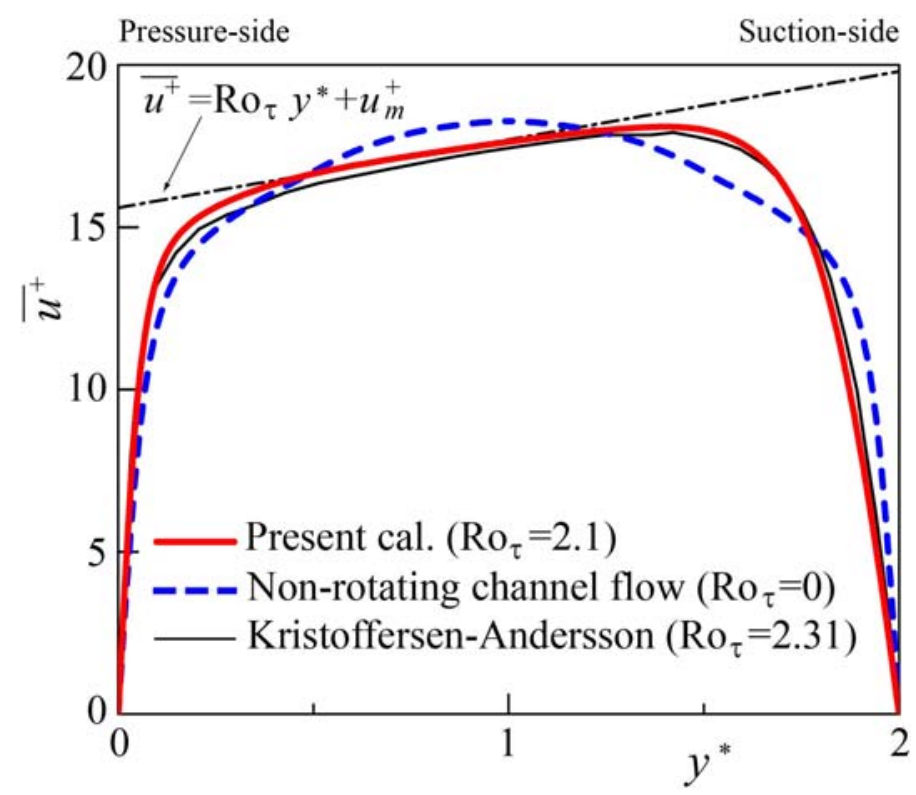

Fig. (5). Mean velocity profile in global coordinates.

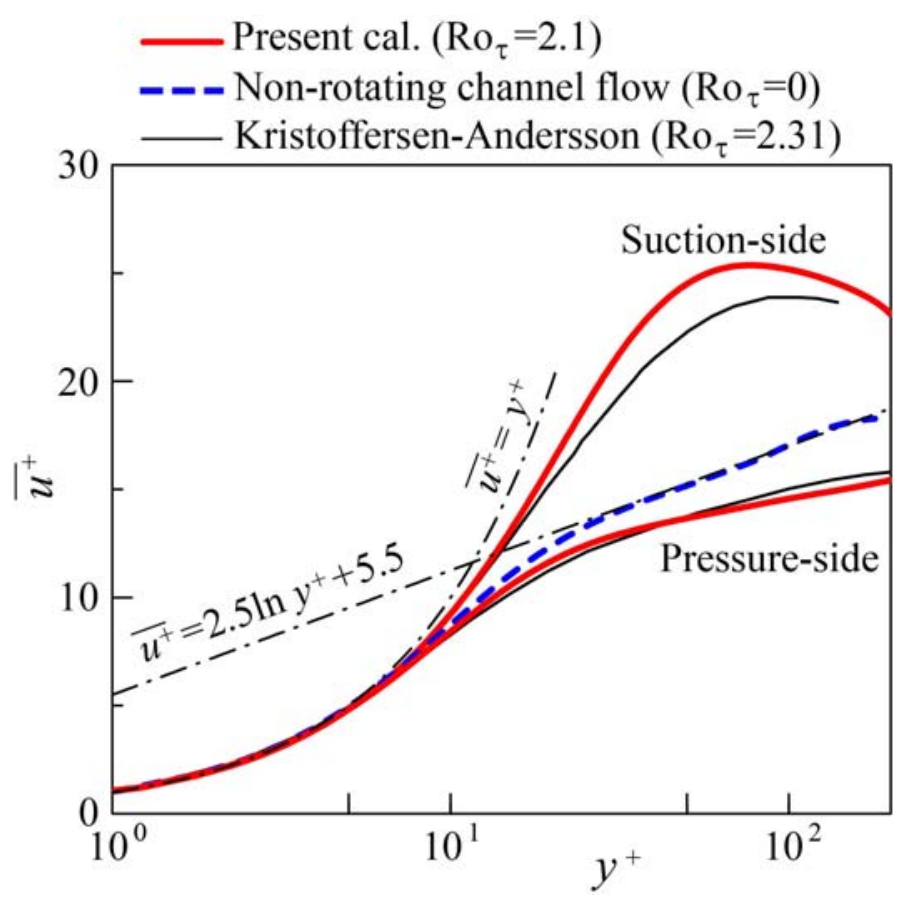

Fig. (6). Mean velocity profile in wall coordinates.

the suction-wall $\left(y^{*}=2\right)$. The velocity gradient remains unaltered at the mid-channel, being parallel with the nondimensional rotation number $\mathrm{Ro}_{\tau}$.

The mean velocity $\overline{u^{+}}$changes as a function of wall coordinate as plotted in Fig. (6), where the velocities are expressed in the nondimensional form with the use of the corresponding friction velocities $u_{\tau p}$ and $u_{\tau s}$. The logarithmic region disappears near the suction-wall, and the velocity distribution almost indicates a feature for laminar flow. The velocity near the pressure-wall is lower than that in the nonrotating channel at the logarithmic region.

The Reynolds shear stress $-\overline{u^{\prime+} v^{\prime+}}$ distributes as shown in Fig. (7). It is heightened by the channel rotation near the pressure-wall, while it is lowered near the suction-wall. The gradient at the mid-channel coincides with that in the nonrotating channel.

Fig. (8) shows the turbulence intensities. The channel rotation increases the turbulent intensities near the pressurewall, but it decreases them near the suction-wall. These are 


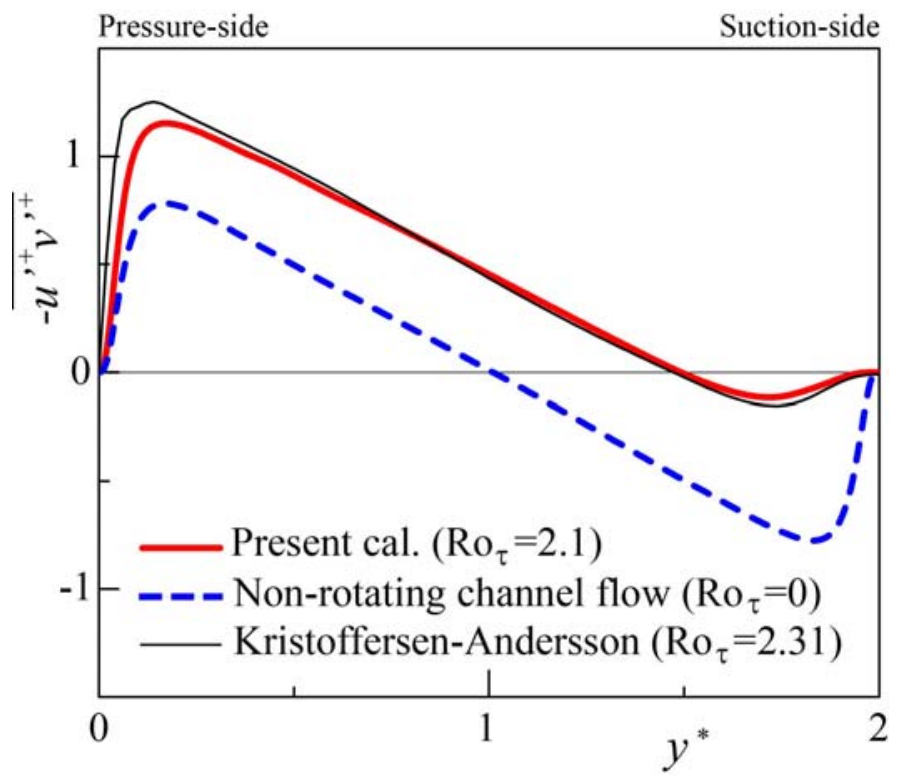

Fig. (7). Reynolds shear stress.
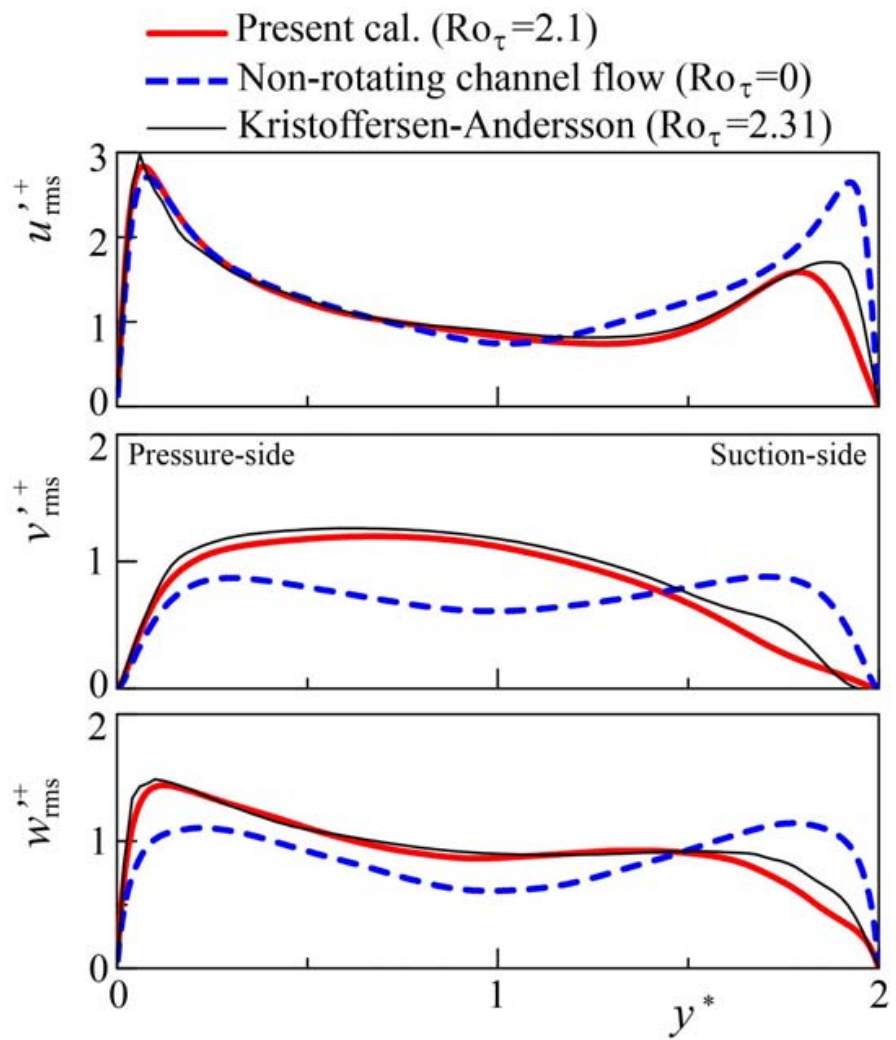

Fig. (8). Rms of velocity fluctuation.

attributable to the changes in the Reynolds shear stress shown in Fig. (7).

The abovementioned results agree nearly with the DNS at $\operatorname{Re}_{\tau}=194$ and $\mathrm{Ro}_{\tau}=2.31$ of Kristoffersen and Andersson [12], demonstrating the validity of the DNS for the rotating turbulent channel flows by the authors' VIC method.

The two-point correlations of the streamwise velocity fluctuation $u^{\prime}, R_{u u}$, are presented in Fig. (9), where the re- sults near the pressure- and suction-walls are plotted. Fig. (9a) shows the change in the streamwise direction. The channel rotation slightly decreases $R_{u u}$ near the pressurewall. The slight reduction of the fluctuation scale occurs there. Near the suction-wall, the decrement of $R_{u u}$ in the streamwise direction becomes gradual due to the channel rotation. One can reconfirm that the flow exhibits the laminar characteristics, which is found from the mean velocity profile shown in Fig. (6). The spanwise change in $R_{u u}$ is 


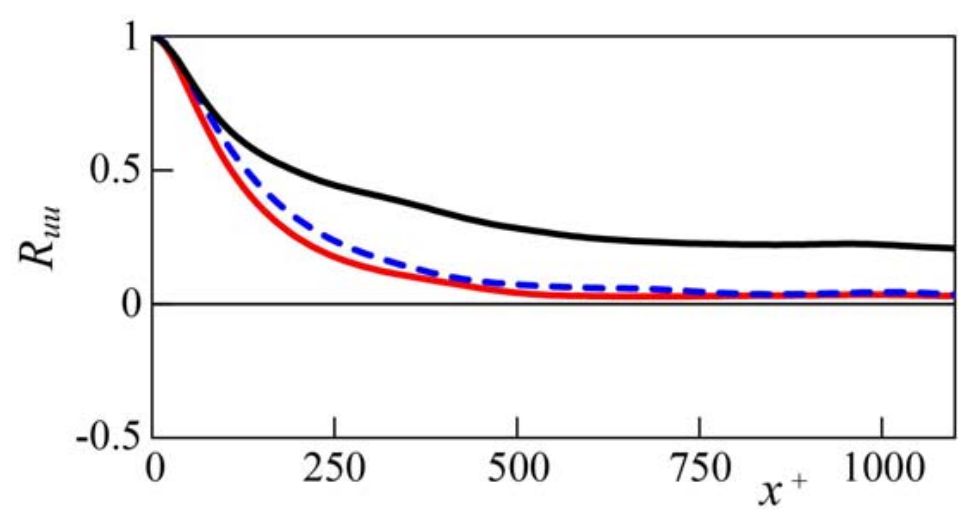

(a) Streamwise

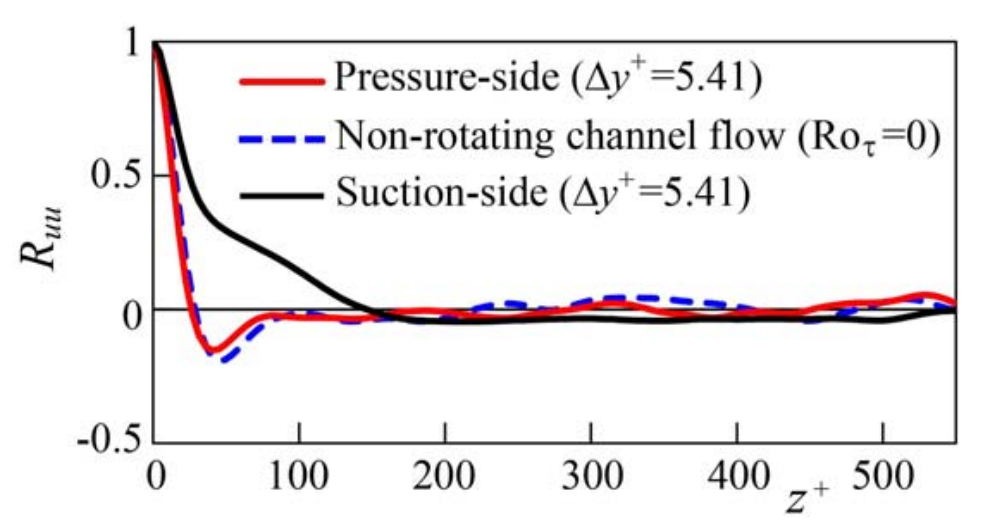

(b) Spanwise

Fig. (9). Two-point correlation of $u^{\prime}$.

plotted in Fig. (9b). For the non-rotating channel flow $\left(\mathrm{Ro}_{\tau}=0\right), R_{u u}$ takes the negative peak at $z^{+}=50$. This is the reflection of the capture for the streak structures [5]. Near the pressure-wall of the rotating channel, the negative peak still appears, and therefore the channel rotation less affects $R_{u u}$. But such negative peak disappears near the suctionwall. This suggests that the flow is laminarized and that the streaks are diminished.

\subsection{Organized Flow Structures}

When calculating the fluctuating component for the streamwise velocity $u^{\prime+}$, the instantaneous iso-surfaces for $u^{\prime+}= \pm 3$ distribute as plotted in Fig. (10). The iso-surface for the second invariant of velocity gradient tensor $Q$, $Q^{+}=0.015$, is also presented in Fig. (10). The iso-surfaces between the central cross-section at $y^{*}=1$ and the walls are projected onto the $x-z$ plane. Fig. (10a) shows the results for the non-rotating flow $\left(\mathrm{Ro}_{\tau}=0\right)$. The high-speed streak $\left(u^{\prime+}=3\right)$ and the low-speed streak $\left(u^{1+}=-3\right)$ appear reciprocally in the spanwise $(z)$ direction. The streak structures are favorably resolved. The streamwise vortices are vividly visualized as found from the distribution of $Q$. The results for the rotating flow $\left(\mathrm{Ro}_{\tau}=2.1\right)$ are plotted in Figs. (10b) and $(10 \mathrm{c})$. As the turbulent intensity decreases due to the reduction of the Reynolds shear stress (see Fig. 7), the streaks are almost diminished as shown in Fig. (10b). But the effect of channel rotation less appears near the pressureside as found in Fig. (10c).

Fig. (11) is the perspective view of the streaks and the streamwise vortices presented in Fig. (10). Near the suctionwall, the organized vortical structures, which are the distinctive features of the turbulent channel flow, almost disappear.

Fig. (12) shows the iso-surfaces for $u^{\prime+}$ and $Q^{+}$projected onto the $z-y$ plane perpendicular to the flow direction. For the non-rotating flow $\left(\mathrm{Ro}_{\tau}=0\right)$, the iso-surfaces concentrate near the walls as found from Fig. (12a). The organized vortical structures appear near the walls. For the rotating flow ( $\operatorname{Ro}_{\tau}=2.1$ ), the streamwise vortices and the low-speed streaks also exist locally at the mid-channel as shown in Fig. (12b). This is because a secondary flow of the TaylorGörtler vortex pattern transports the momentum near the suction-wall to the mid-channel region as discussed later.

On a cross-section perpendicular to the flow direction, the velocity distributes as shown in Fig. (13), where the distribution on the $z-y$ plane of $x^{+}=0$ at the same time point as Fig. (12b) is presented. Some large-scale eddies are observed in the region at a distance from the pressure-wall. 


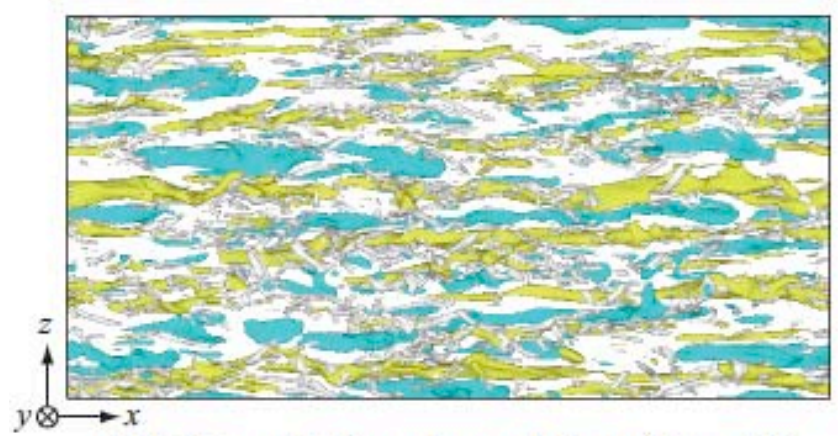

(a) Non-rotating channel flow $\left(\mathrm{Ro}_{\tau}=0\right)$

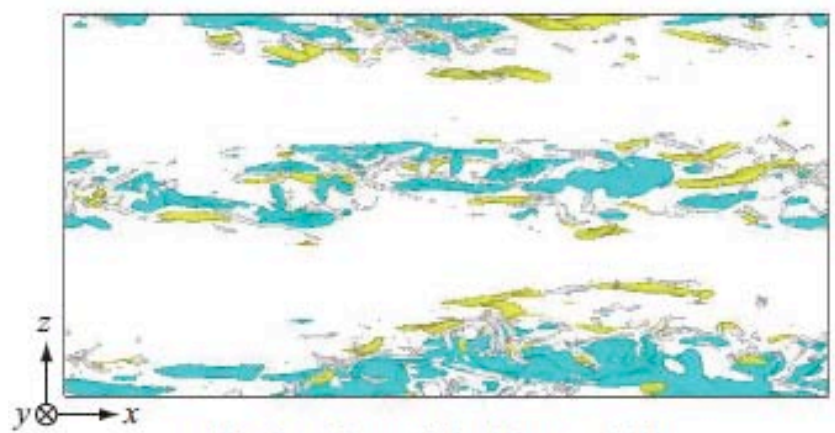

(b) Suction-side $\left(\mathrm{Ro}_{\tau}=2.1\right)$

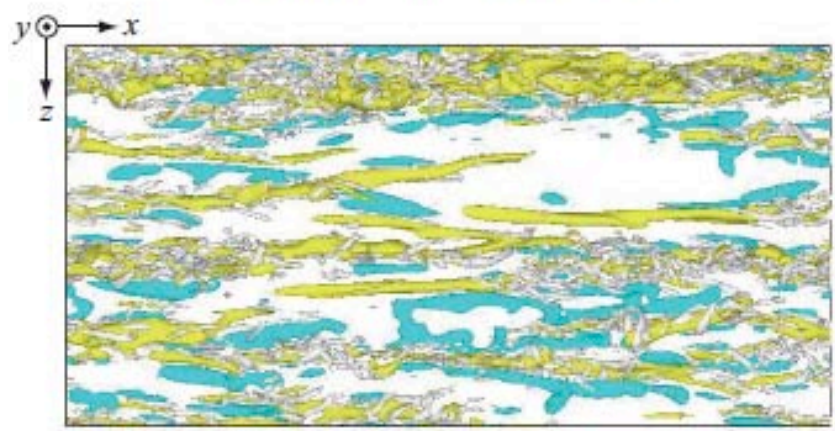

(c) Pressure-side $\left(\mathrm{Ro}_{\tau}=2.1\right)$

Fig. (10). Visualization of high- and low-speed streaks and second invariant of velocity gradient tensor $Q\left(u^{\prime+}=3\right.$; blue, $u^{\prime+}=-3$; yellow, $Q^{+}=0.015$; light-gray).

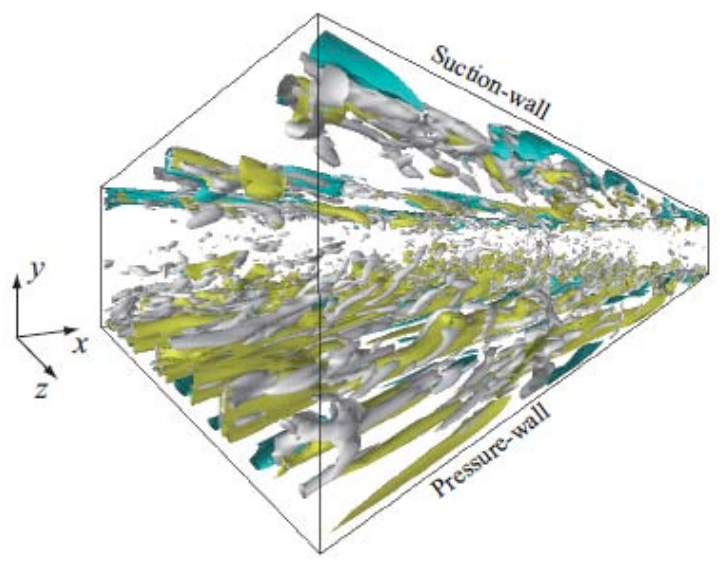

Fig. (11). Perspective view of high- and low-speed streaks and second invariant of velocity gradient tensor $\left(u^{\prime+}=3\right.$; blue, $u^{\prime+}=-3$; yellow, $Q^{+}=0.015$; light-gray). 


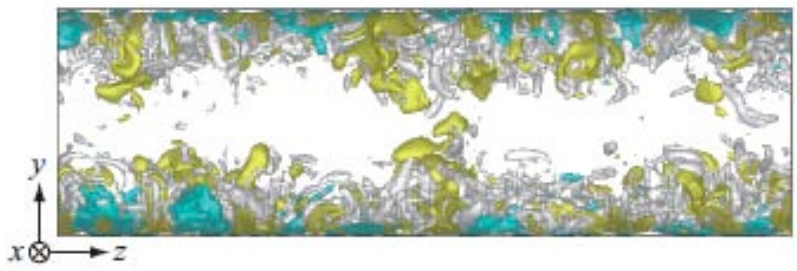

(a) Non-rotating channel flow $\left(\mathrm{Ro}_{\tau}=0\right)$

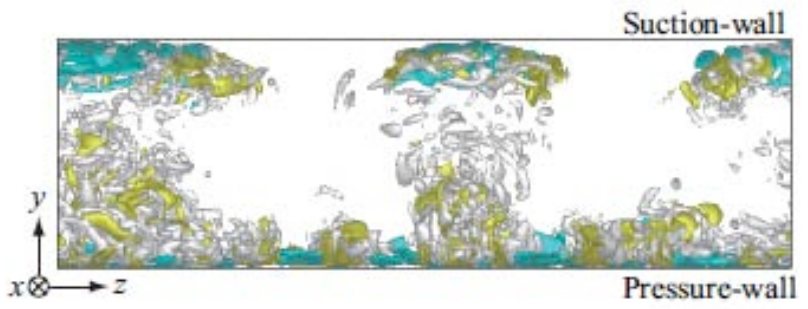

(b) Rotating channel $\left(\mathrm{Ro}_{\tau}=2.1\right)$

Fig. (12). Iso-surfaces of high- and low-speed streaks and second invariant of velocity gradient tensor $Q$ projected onto $z-y$ plane $\left(u^{\prime+}=3\right.$; blue, $u^{\prime+}=-3$; yellow, $Q^{+}=0.015$; light-gray).

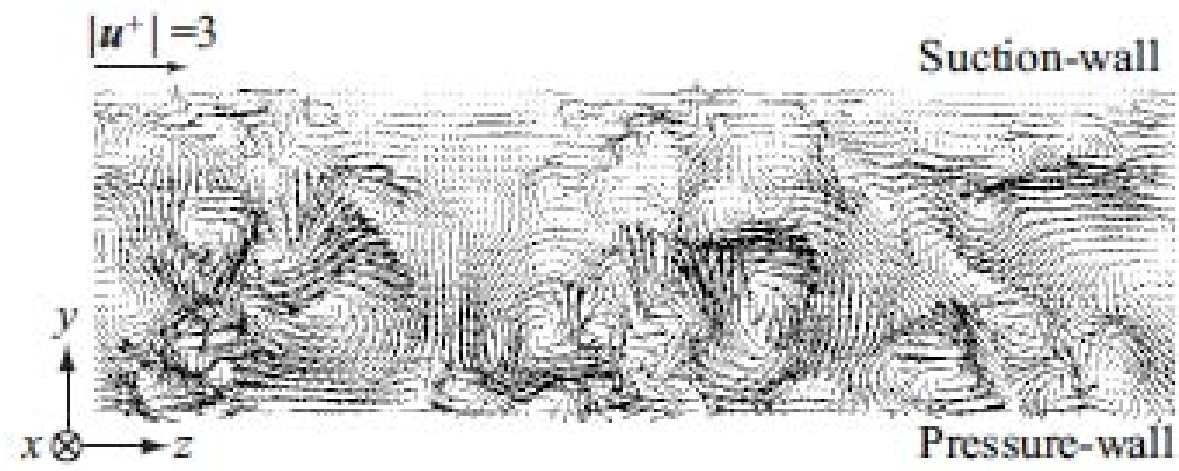

Fig. (13). Velocity distribution on $z-y$ section.

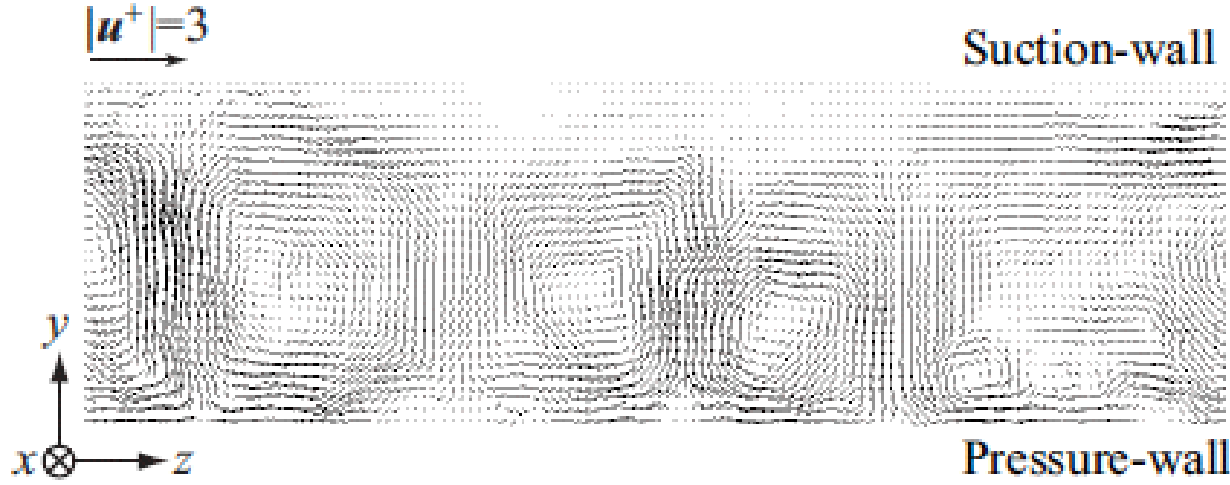

Fig. (14). Time-averaged velocity distribution on $z-y$ section.

However, the high-speed flow is not found near the suctionwall.

Calculating the time-averaged flow velocity during $5.6 \delta / u_{\tau}$ on the cross-section depicted in Fig. (13), it distributes as shown in Fig. (14). Two pairs of vortices exist, and the center of each vortex locates closer to the pressure- wall. These correspond to the secondary flow of the TaylorGörtler vortex pattern, which were explored through the experiments of Johnston et al. [6] and Koyama et al. [8], the LES of Miyake and Kajishima [10], and the DNS of Kristoffersen and Andersson [12]. Kristoffersen and Andersson [12] grasped two pairs of vortices by the DNS of $L_{z} / L_{y}=\pi$, 
$\operatorname{Re}_{\tau}=194$ and $\operatorname{Ro}_{\tau}=2.31$. These computational conditions are almost the same as this study. Therefore, it is confirmed that the present simulation can resolve accurately the secondary flow of the Taylor-Görtler vortex pattern.

\section{CONCLUSIONS}

The DNS for an incompressible fully-developed turbulent flow between two infinite parallel plates in spanwise rotation with a constant angular velocity $\Omega$ is conducted. The vortex in cell (VIC) method, of which computational accuracy was heightened by the authors in their prior study, is employed for the DNS. The Reynolds number based on the friction velocity $u_{\tau}$ and the channel half width $\delta$, $u_{\tau} \delta / v$, is 171 , and the nondimensional rotation number $2 \Omega \delta / u_{\tau}$ is 2.1 .

The DNS shows that the effects of the channel rotation on the turbulence statistics, such as the mean velocity and the Reynolds shear stress, are favorably analyzed. It also indicates that the disappearance of the streak structures near the suction-wall due to the channel rotation and the secondary flow of the Taylor-Görtler vortex pattern are successfully captured. These demonstrate the validity of the DNS for rotating turbulent channel flows by the authors' VIC method.

\section{NOMENCLATURES}

\begin{tabular}{|c|c|c|}
\hline$a$ & $=$ & side length of cubic cavity \\
\hline$h$ & $=$ & grid width \\
\hline$L$ & $=$ & side length of computational domain \\
\hline$N$ & $=$ & number of grids along one axis \\
\hline$Q$ & $=$ & second invariant of velocity gradient tensor \\
\hline $\operatorname{Re}_{\tau}$ & $=$ & Reynolds number $=u_{\tau} \delta / v$ \\
\hline $\operatorname{Ro}_{\tau}$ & $=$ & nondimensional rotation number $=2 \Omega \delta / u_{\tau}$ \\
\hline$R_{u u}$ & $=$ & $\begin{array}{l}\text { two-point correlation coefficient of stream- } \\
\text { wise velocity fluctuation }\end{array}$ \\
\hline$t$ & $=$ & time \\
\hline $\boldsymbol{u}$ & $=$ & velocity \\
\hline$u, v, w$ & $=$ & velocity components in $x, y, z$ directions \\
\hline$u_{m}$ & $=$ & bulk mean velocity \\
\hline$u_{\tau}$ & $=$ & friction velocity $=\left(\bar{\tau}_{w} / \rho\right)^{1 / 2}$ \\
\hline$W$ & $=$ & redistribution function of vorticity \\
\hline$x$ & $=$ & position vector \\
\hline$x, y, z$ & $=$ & orthogonal coordinates \\
\hline$\delta$ & $=$ & channel half width \\
\hline$\Delta t$ & $=$ & time increment \\
\hline
\end{tabular}

$\begin{array}{lll}v & = & \text { kinematic viscosity } \\ \rho & = & \text { density } \\ \tau_{w} & = & \text { wall shear stress } \\ \phi & = & \text { scalar potential } \\ \psi & = & \text { vector potential } \\ \Omega & = & \text { rotating angular velocity of channel } \\ \omega & = & \text { vorticity }=\nabla \times \mathbf{u}\end{array}$

\section{Subscripts}

$=$ non-rotating channel $(\Omega=0)$

$p \quad=\quad$ pressure-wall

rms $=$ root mean square

$S \quad=\quad$ suction-wall

$x, y, z=$ components in directions $x, y$ and $z$

$+\quad=$ normalized by $u_{\tau}, v$ and $\rho$

$* \quad=$ normalized by $\delta$

- $\quad$ fluctuation component

$-\quad=\quad$ statistically averaged over $x, z$ and $t$

\section{CONFLICT OF INTEREST}

The authors confirm that this article content has no conflicts of interest.

\section{ACKNOWLEDGEMENTS}

Declared none.

\section{REFERENCES}

[1] I. P. Christiansen, "Numerical simulation of hydrodynamics by the method of point vortices," J. Comput. Phys., vol. 13, pp. 363-379, 1973.

[2] G.-H. Cottet and P. Poncet, "Advances in direct numerical simulations of 3D wall-bounded flows by Vortex-in-Cell methods", $J$. Comput. Phys., vol. 193, pp. 136-158, 2003.

[3] R. Cocle, G. Winckelmans, and G. Daeninck, "Combining the vortex-in-cell and parallel fast multipole methods for efficient domain decomposition simulations", J. Comput. Phys., vol. 227, pp. 9091-9120, 2008.

[4] P. Chatelain, A. Curioni, M. Bergdorf, D. Rossinelli, W. Andreoni, and P. Koumoutsakos, "Billion vortex particle direct numerical simulations of aircraft wakes", Comput. Methods Appl Mech. Eng., vol. 197, pp. 1296-1304, 2008.

[5] T. Uchiyama, Y. Yoshii, and H. Hamada, "Direct numerical simulation of a turbulent channel flow by an improved vortex in cell method", Int. J. Numer. Methods Heat Fluid Flow, (accepted and in press).

[6] J. P. Johnston, R. M. Halleen, and D. K. Lezius, "Effects of spanwise rotation on the structure of two-dimensional fully developed turbulent channel flow", J. Fluid Mech., vol. 56, pp. 533-557, 1972.

[7] P. Rothe and J. P. Johnston, "Free shear layer behavior in rotating systems", Trans. ASME, J. Fluid Eng., vol. 101, pp. 117-120, 1979.

[8] H. Koyama, S. Masuda, I. Ariga, and I. Watanabe, "Stabilizing and destabilizing effects of Coriolis force on two-dimensional laminar and turbulent boundary layers", Trans. ASME, J. Eng. Power, vol. 101, pp. 23-31, 1979. 
[9] K. Kikuyama and M. Murakami, "Flows and pressure recovery in a rotating diffuser (Effects of inlet velocity distributions)", JSME, Bull., vol. 26, pp. 970-977, 1983.

[10] Y. Miyake and T. Kajishima, "Numerical simulation of the effects of Coriolis force on the structure of turbulence. I - Global effects", JSME, Bull., vol. 29, pp. 3341-3346, 1986.

[11] E. Lamballais, O. Metais and M. Lesieur, "Spectral-dynamical model for large-eddy simulations of turbulent rotating channel flow", Theor Comput Fluid Dyn, vol. 12, pp. 149-177, 1998.
[12] R. Kristoffersen and H. Andersson, "Direct simulation of lowReynolds-number turbulent flow in a rotating channel", J. Fluid Mech., vol. 256, pp. 163-197, 1993.

[13] O. Grundestam, S. Wallin, and V. Johansson, "Direct numerical simulations of rotating turbulent channel flow", J. Fluid Mech., vol. 598, pp. 177-199, 2008.

[14] G.-H. Cottet and P. D. Koumoutsakos, Vortex Methods: Theory and Practice, Cambridge University Press, 2000.

[15] J. Kim, P. Moin, and R. Moser, "Turbulence statistics in fully developed channel flow at low Reynolds number", J. Fluid Mech., vol. 177, pp. 133-166, 1987.

Received: August 16, 2013

Revised: September 12,2013

Accepted: October 15, 2013

(C) Praveen and Savitha; Licensee Bentham Open.

This is an open access article licensed under the terms of the Creative Commons Attribution Non-Commercial License (http://creativecommons.org/licenses/by-nc/3.0/) which permits unrestricted, non-commercial use, distribution and reproduction in any medium, provided the work is properly cited. 